$\underline{\text { Supporting Information }}$

\title{
Frequency Shifts in SERS-Based Immunoassays: Mechanistic Insights and Application in Protein Carbonylation Detection
}

Hao Ma, ${ }^{1}$ Songlin Liu, ${ }^{1}$ Naiqing Zheng, ${ }^{2}$ Yawen Liu, ${ }^{1}$ Xiao Xia Han, ${ }^{1}$ Chengyan He, ${ }^{3}$ Hui $\mathrm{Lu}^{4 *}$ and Bing $\mathrm{Zhao}^{1^{*}}$

1. State Key Laboratory of Supramolecular Structure and Materials, Jilin University, Changchun 130012, P. R. China.

2. National Engineering Laboratory for AIDS Vaccine, School of Life Science, Jilin University, Changchun 130012, P. R. China

3. China-Japan Union Hospital of Jilin University, Changchun 130033, P. R. China.

4. School of Biological Sciences, Faculty of Biology, Medicine and Health, The University of Manchester, Manchester M13 9PT, UK.

Corresponding Author: *Hui.Lu@manchester.ac.uk (H.L.); zhaob@jlu.edu.cn (B.Z.)

\section{Table of Contents}

Supplementary experimental details:

Materials; Preparation of self-assemble chips; preparation of antibody capture chips; SERS characterization and analysis; preparation and determination of carbonyl protein; immunoassay protocol; computational details.

\section{Supplementary Tables}

Table S1. Comparation of calculated dipole moment based on different functionals and methods.

Table S2. . Relevant assays for carbonylated proteins.

\section{Supplementary Figures.}

Figure S1. Energy gaps between HOMO and LUMO with different solvents. The ground state is stabilized by the polarization of solvent.

Figure S2. SERS spectra of the MBA -Ag chips with different solvents: a. $\mathrm{H}_{2} \mathrm{O}$, b. DMSO, c. $\mathrm{MeCN}$, d. $\mathrm{C}_{2} \mathrm{H}_{5} \mathrm{OH}$, e. $\mathrm{CCl}_{4}$, f. No solvent; (B) zoomed-in image of the dotted line in (A).

Figure S3. SERS spectra of the Ag-MBA-lgE chips with different antigens; (B) Zoomed-in image of the dotted line in (A).

Figure S4. UV-Vis spectra of DNP-BSA (red), the blank (blue, BSA) and the control (blank, DNPH). 


\section{Experimental details}

\section{Materials.}

MBA, bovine serum albumin (BSA), Glycine (Gly), Monoclonal Anti-Dinitrophenyl antibody IgE isotype produced in mouse (D8406), Anti-DNP antibody produced in goat (D9781) $\mathrm{AgNO}_{3}$, and poly(diallyldimethylammonium chloride) ( $\mathrm{Mw}=$ 200,000-350,000, $20 \mathrm{wt} \%$ aqueous solution) were obtained from Sigma-Aldrich. 2,4-Dinitrophenylhydrazine $\quad$ (DNPH), 1-(3-dimethylaminopropyl)3-ethylcarbodiimide hydrochloride (EDC), and N-hydroxysuccinimide (NHS) were obtained from J\&K Chemical Co. Alpha-fetoprotein (AFP) was obtained from chemiluminescence kit purchased from Beckman Coulter (Product Name: AFP (300 Test Kit); Part Number: 33211; Series Name: ACCESS $\left.{ }^{\circledR}\right)$. The phosphate buffered saline (PBS; $0.01 \mathrm{M}, \mathrm{pH} 7.2$ ) used in this study contained $0.8 \% \mathrm{NaCl}, 0.02 \% \mathrm{KH}_{2} \mathrm{PO}_{4}$, $0.02 \% \mathrm{KCl}$, and $0.12 \% \mathrm{Na}_{2} \mathrm{HPO}_{4} \cdot 12 \mathrm{H}_{2} \mathrm{O}$. All chemicals were analytical-grade reagents and used without further purification. Milli-Q water was used in the study.

\section{Preparation of self-assemble chips}

Ag hydrosol was synthesized by a conventional synthetic route reported by Lee and Meisel. In brief, $36 \mathrm{mg}$ of $\mathrm{AgNO}_{3}$ was dissolved in $200 \mathrm{~mL}$ of water $(1.0 \mathrm{mM}$ aqueous), and then the solution was heated to $98{ }^{\circ} \mathrm{C}$ with rapid stirring under reflux. A $4 \mathrm{~mL}$ solution of $1 \%$ sodium citrate was added to the solution, and the solution was boiled for $40 \mathrm{~min}$. The Ag nanoparticles with the diameter of 60-70 $\mathrm{nm}$ were prepared. The gold hydrosol was also synthesized by means of same method. Subsequently, glass/silicon slides were immersed in a boiling solution prepared by mixing $30 \%$ $\mathrm{H}_{2} \mathrm{O}_{2}$ and $98 \% \mathrm{H}_{2} \mathrm{SO}_{4}$ with a volume ratio of $3: 7$. After rinsing, the hydroxylated glass/silicon slides were obtained. Then they were soaked in a $0.5 \%$ PDDA solution for $40 \mathrm{~min}$. After repeated rinsing with water and drying with nitrogen gas, the PDDA-coated slides were soaked in silver hydrosol for $4 \mathrm{~h}$. Eventually, a layer of Ag NPs was assembled on the glass surface by electrostatic interaction.

The glass/silicon chips were subsequently immersed into an MBA aqueous solution $\left(10^{-4} \mathrm{M}\right)$ for $12 \mathrm{~h}$. The chips were rinsed three times with Milli-Q water and dried with nitrogen gas.

\section{Preparation of the antibody capture chips}

The glass/silicon chips were immersed in an aqueous solution of $0.1 \mathrm{M}$ EDC/NHS for $3 \mathrm{~h}$, and then the chips were rinsed three times with Milli-Q water and dried with nitrogen gas. The chips were stored in the plastic centrifuge tube under a nitrogen atmosphere. Subsequently, antibodies were immobilized by pipetting $500 \mu \mathrm{L}$ of the protein solution onto chips in a plastic centrifuge tube. The reaction was allowed to progress overnight at $4{ }^{\circ} \mathrm{C}$ or for $4 \mathrm{~h}$ at $37{ }^{\circ} \mathrm{C}$. Subsequently, the chips were rinsed with PBS solution and gently dried with nitrogen gas. The blocking buffer $(0.1 \mathrm{mg} / \mathrm{L}$ BSA and $0.1 \mathrm{mg} / \mathrm{mL}$ Glycine in PBS, $\mathrm{pH}$ 7.4) was pipetted onto the chips and incubated for $2 \mathrm{~h}$. (Note that 2,4-DNPH also reacts with activated carbonyl groups. This is the reason why we introduce glycine into blocking buffer.) The chips were then rinsed three times with PBS and stored in the plastic centrifuge tube under a nitrogen atmosphere. 


\section{SERS characterization and Analysis}

All SERS spectra were measured on a Jobin Yvon/HORIBA LabRam ARAMIS Raman spectrometer equipped with an HeNe Laser $(632.8 \mathrm{~nm})$. The typical exposure time for each chip was $1 \mathrm{~s}$ with one-time accumulation with a $0.3 \mathrm{D}$ filter. The grating scale is $18001 \mathrm{~mm}^{-1}$, the spectra resolution is $1.6 \mathrm{~cm}^{-1}$. And the spectra sampling distance is $0.5 \mathrm{~cm}^{-1}$.

\section{Preparation and determination of carbonyl protein ${ }^{[1]}$}

BSA at concentration of $5 \mathrm{mg} / \mathrm{mL}$ was used as a model to obtain oxidazed protein. Briefly, $10 \mathrm{~mL}$ protein solution was mixed with $10 \mathrm{ml}$ of $0.5 \% \mathrm{H}_{2} \mathrm{O}_{2}$ and incubated for $3 \mathrm{~h}$ at room temperature. Excess $\mathrm{H}_{2} \mathrm{O}_{2}$ in oxiBSA was removed by introducing $50 \mu \mathrm{l}$ of $10 \mu \mathrm{M} \mathrm{Fe}_{2}\left(\mathrm{SO}_{4}\right)_{3}$. Then, $1.5 \mathrm{ml}$ of $10 \mathrm{mM}$ 2,4-DNPH in $2 \mathrm{M} \mathrm{HCl} \mathrm{DNPH}$ was added into $10 \mathrm{ml}$ oxiBSA solutions. After incubation of the protein solutions for $60 \mathrm{~min}$ in the dark at room temperature, $4 \mathrm{~mL}$ of the solution was concentrated by ultrafiltration centrifugation at $5000 \mathrm{rpm}$ for 10 minutes to $400 \mu \mathrm{l}$ and washed 2 times to remove excess DNPH. Concentrated solution of DNPH-oxiBSA (about $300 \mu \mathrm{L}$ ) was diluted in $4 \mathrm{ml}$ deionized $\mathrm{H}_{2} \mathrm{O}$ to be measured. The initial concentration of carbonylated protein is based on the classic method. The approach involves the reaction between the proteins and DNPH followed by the spectrophotometric quantification of the acid hydrazones at $366 \mathrm{~nm}$. As shown in Figure S4, the carbonyl contents can be determined from the absorbance of DNP-BSA at $366 \mathrm{~nm}(\mathrm{~A}=0.316)$, which is calculated to be $14.36 \mathrm{nmol} / \mathrm{mL}$ with a molar absorption coefficient of $22000 \mathrm{M}^{-1} \mathrm{~cm}^{-1}$.

\section{Immunoassay protocol}

Subsequently, the proteins were immobilized by pipetting $10 \mu \mathrm{L}$ of proteins solution at different concentrations onto chips. Note that the $\mathrm{pH}$ of the proteins is very low because of $\mathrm{HCl}$, which we control the volume of proteins within $20 \mu \mathrm{L}$. It is within the buffer capacity of $500 \mu \mathrm{L}$ PBS. The low $\mathrm{pH}$ also influence the frequency shift of MBA, which has been reported by several groups. The reaction was allowed to progress overnight at $4{ }^{\circ} \mathrm{C}$ or for $4 \mathrm{~h}$ at $37{ }^{\circ} \mathrm{C}$. The chips were rinsed three times with PBS and stored in the plastic centrifuge tube under a nitrogen atmosphere.

\section{Computational details}

All quantum chemical calculations were carried out using the Gaussian 09 program. ${ }^{\text {[2] }}$ The geometries of $\mathrm{Ag}_{3}$-MBA were optimized by DFT using the functional of M062X, an acceptable functionals for calculating solvent effect. The solvent model density (SMD) continuum solvation model was introduced. At this geometry, the frequency calculation was obtained. And electronic transitions were calculated with time-dependent density functional theory (TD-DFT), which only singlet-singlet excitations were evaluated. The basis set of proposed model was a combination of 6-311+G* for $\mathrm{C}, \mathrm{S}, \mathrm{O}$, and $\mathrm{H}$ atoms and LanL2DZ for Ag atoms. However, it is known to all that corrected parameters of M062X are optimized based on organic molecules. It is not known how accurate the M062X calculation is with respect to transition metals. Hence, we compared the results of dipole moment calculated at PBE0/6-311+G* level with those from M062X. 
As shown in Table S1, comparing with results of PBE0/def2-TZVP, it is obvious that $\mathrm{M} 062 \mathrm{X}$ is overestimated about ground state as well as PBE0/6-311+G*. However, it is interesting to deduce the results for excited states may be more accurate than PBE0/6-311+G*, because it contains higher HF component (0.5). It demonstrates that, although M062X is optimized with organic molecules, it also reveals acceptable accuracy, which is suitable for qualitative calculation in transition metal systems.

Table S1. Comparation of calculated dipole moment based on different functionals and methods.

\begin{tabular}{ccccccc}
\hline & \multicolumn{2}{c}{ M062X/6-311+G* } & \multicolumn{2}{c}{ PBE0/6-311+G* } & \multicolumn{2}{c}{ PBE0/def2-TZVP } \\
\hline Solvent & HOMO & LUMO & HOMO & LUMO & HOMO & LUMO \\
None & 11.95 & 1.97 & 11.35 & 2.12 & 10.44 & $*$ \\
$\mathrm{H}_{2} \mathrm{O}$ & 18.86 & 17.26 & 18.58 & 17.49 & 17.64 & $*$ \\
$\mathrm{DMSO}$ & 18.20 & 16.51 & 18.04 & 16.98 & 16.89 & $*$ \\
$\mathrm{MeCN}$ & 18.18 & 16.44 & 17.84 & 16.29 & 16.87 & $*$ \\
Ethanol & 18.41 & 16.61 & 18.18 & 16.47 & 17.24 & $*$ \\
$\mathrm{CCl}_{4}$ & 14.84 & 9.97 & 14.56 & 2.99 & 13.58 & $*$ \\
\hline
\end{tabular}

* Absence of derivative for def2-TZVP in Gaussian 09, only dipole moment of HOMO in present system was obtained.

Table S2. Relevant assays for carbonylated proteins.

\begin{tabular}{lllc}
\hline Methods & LOD $(\mathrm{nmol} / \mathrm{mg})$ & Dynamic Range(nmol $/ \mathrm{mg})$ & References \\
\hline SERS & 0.04 & $0.04-3.78$ & This work \\
ELISA & 0.09 & $0.09-1.61$ & 3 \\
Raman & 2.98 & unknown & 4 \\
Western blot & 223 & $223-373$ & 1 \\
Immunoblotting & 0.06 & unknown & 1 \\
HPLC & 30 & unknown & 1 \\
\hline
\end{tabular}




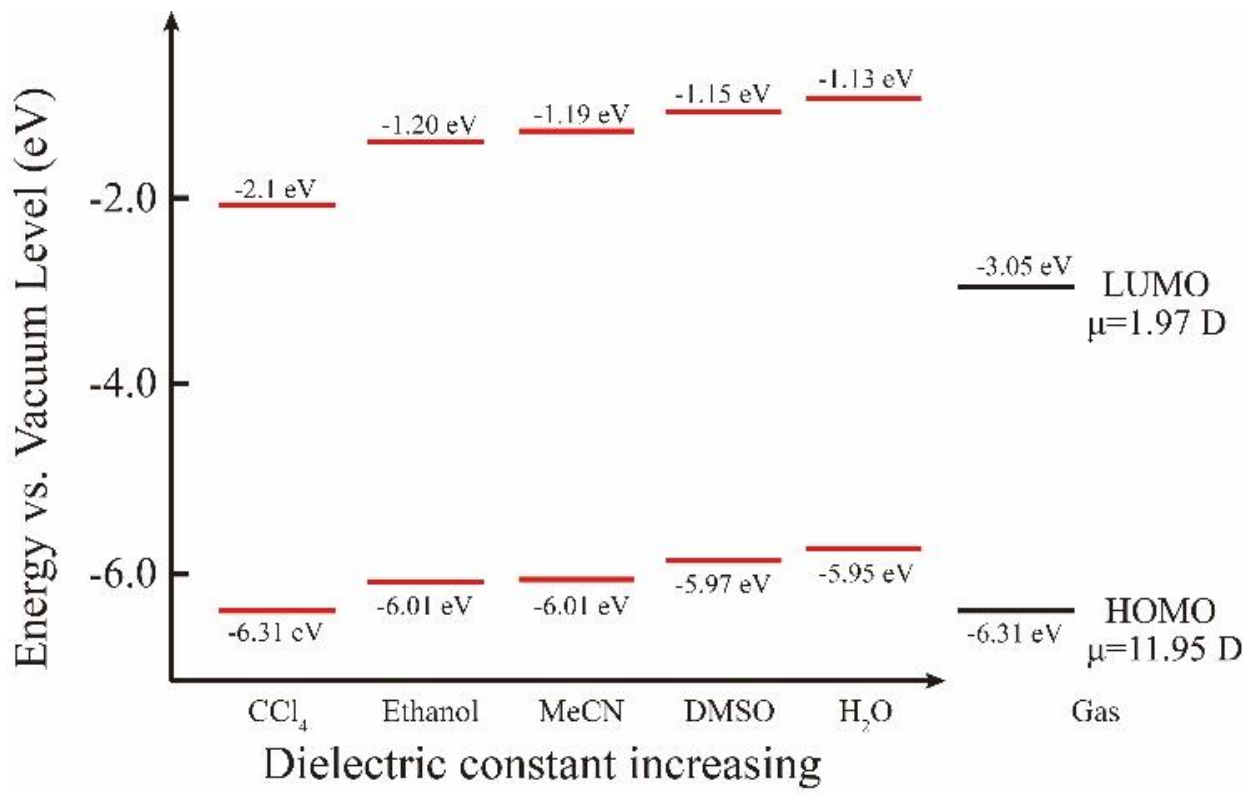

Figure S1. Energy gaps between HOMO and LUMO with different solvents. The ground state is stabilized by the polarization of solvent. 
As a manifestation of CT transition from the Fermi level of Au NPs to the LUMO of MBA, as well as in the Ag-MBA system, the frequency shifts revealed a same tendency as that of $b_{2}$ intensity change. It further demonstrated a direct correction between the CT and the frequency shifts. Comparing with line at $1075.0 \mathrm{~cm}^{-1}$ in the Ag-MBA with that of $1075.6 \mathrm{~cm}^{-1}$ in the case of Au-MBA chips, we demonstrated the influence of SPR on the electric field is neglectable. However, it has dramatic effect on the CT, because of the lower Fermi level of gold. As shown in Figure S2, CT only occurred within the existence of water.
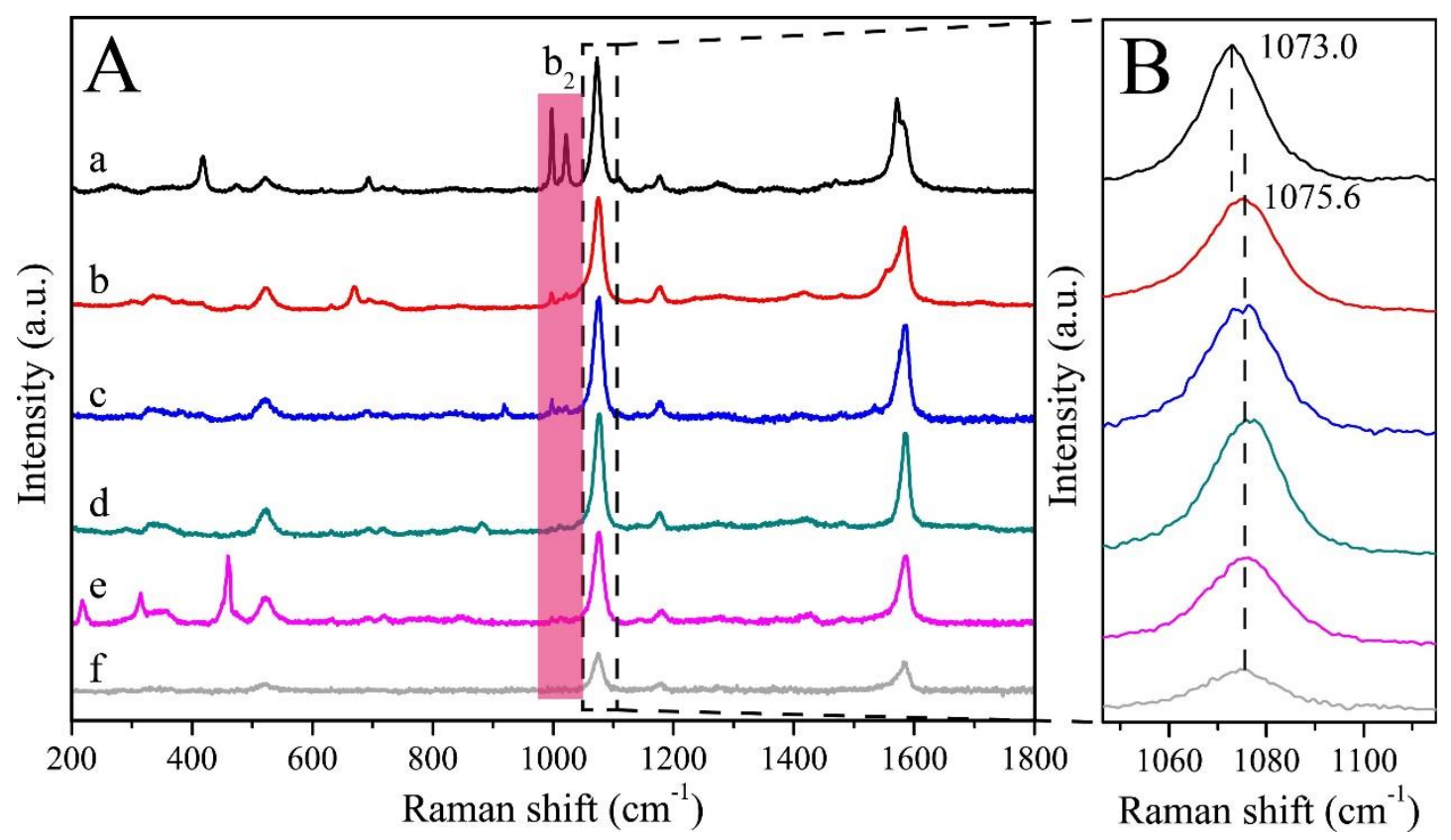

Figure S2. SERS spectra of the MBA-Ag chips with different solvents: $a . \mathrm{H}_{2} \mathrm{O}, \mathrm{b}$. DMSO, c. $\mathrm{MeCN}$, d. $\mathrm{C}_{2} \mathrm{H}_{5} \mathrm{OH}$, e. $\mathrm{CCl}_{4}$, f. No solvent; (B) zoomed-in image of the dotted line in (A). 
As shown in Figure S3, DNPH did not induce a frequency shift, which is consistent with result in the case of IgG. When we introduce the DNP-BSA into Ag-MBA-IgE, we observed a red shift of $1.5 \mathrm{~cm}^{-1}$. Therefore we deduced that the BSA with high molecule weight induced a further deformation of MBA.

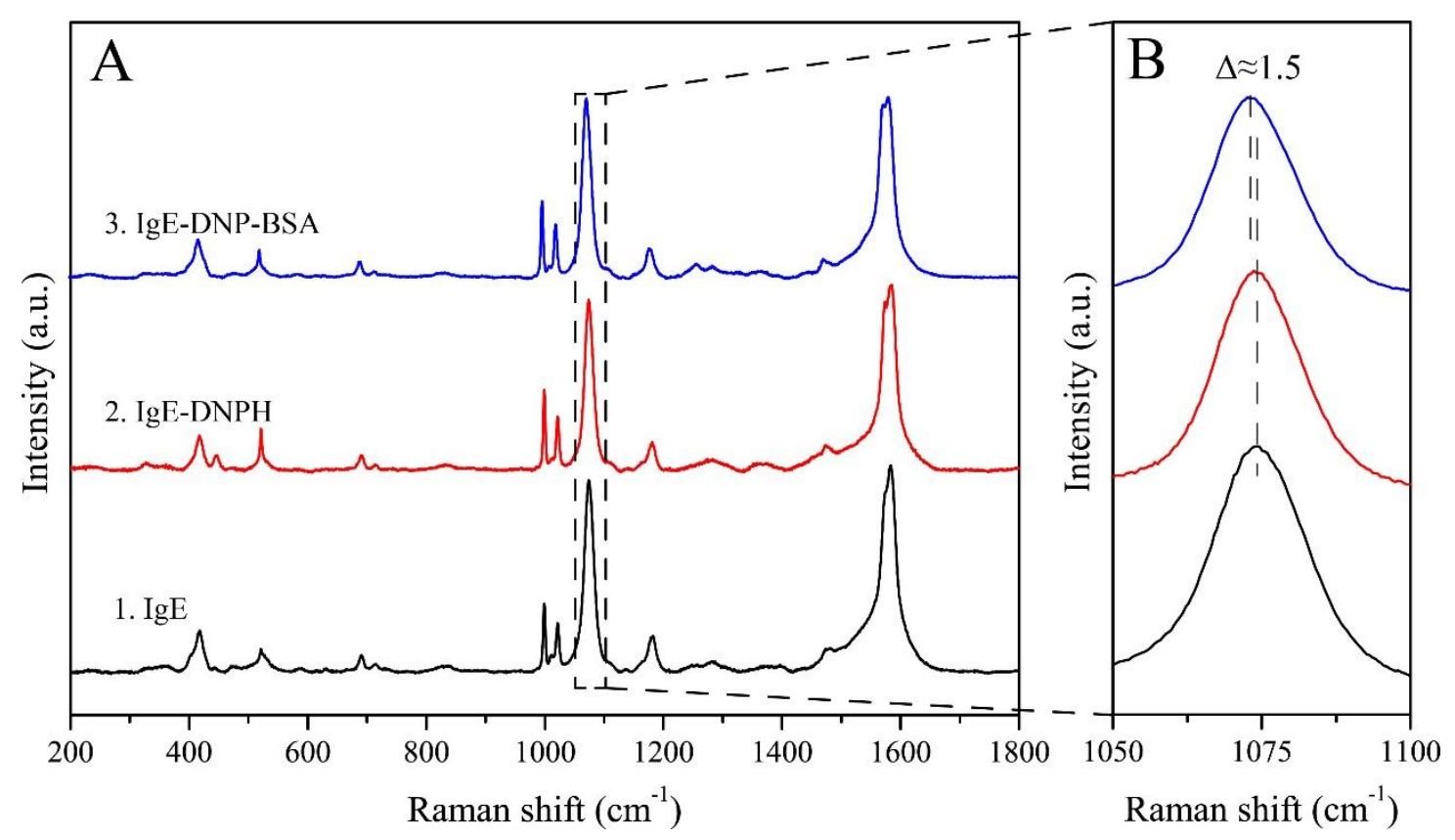

Figure S3. SERS spectra of the Ag-MBA-IgE chips with different antigens; (B) zoomed-in image of the dotted line in (A). 


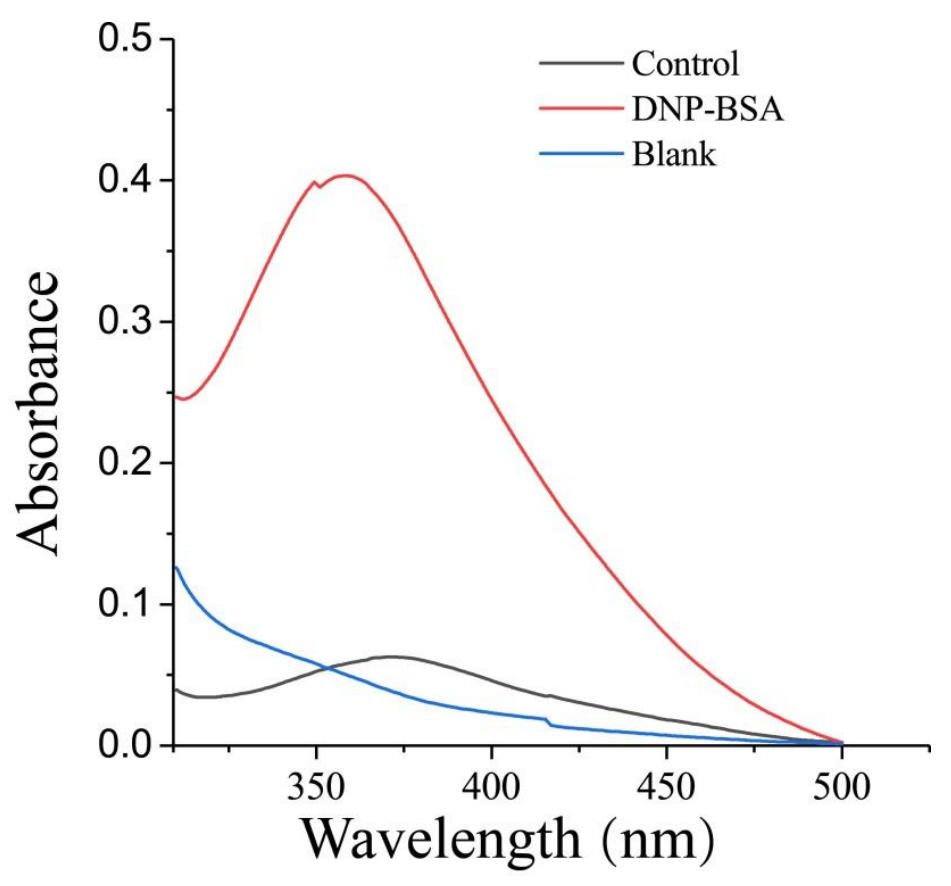

Figure S4. UV-Vis spectra of DNP-BSA (red), the blank (blue, BSA) and the control (blank, DNPH). The absorbance of the DNP-BSA and the control is 0.376 and 0.06 , respectively.

\section{Reference}

[1] I. Dalle-Donne, R. Rossi, D. Giustarini, A. Milzani, R. Colombo., Protein carbonyl groups as biomarkers of oxidative stress, Clinica Chimica Acta, 329 (2003), 23-38

[2] M. J. Frisch, G. W. Trucks, H. B. Schlegel, G. E. Scuseria, M. A. Robb, J. R. Cheeseman, G. Scalmani, V. Barone, B. Mennucci, G. A. Petersson, H. Nakatsuji, M. Caricato, X. Li, H. P. Hratchian, A. F. Izmaylov, J. Bloino, G. Zheng, J. L. Sonnenberg, M. Hada, M. Ehara, K. Toyota, R. Fukuda, J. Hasegawa, M. Ishida, T. Nakajima, Y. Honda, O. Kitao, H. Nakai, T. Vreven, J. A. Montgomery, Jr., J. E. Peralta, F. Ogliaro, M. Bearpark, J. J. Heyd, E. Brothers, K. N. Kudin, V. N. Staroverov, T. Keith, R. Kobayashi, J. Normand, K. Raghavachari, A. Rendell, J. C. Burant, S. S. Iyengar, J. Tomasi, M. Cossi, N. Rega, J. M. Millam, M. Klene, J. E. Knox, J. B. Cross, V. Bakken, C. Adamo, J. Jaramillo, R. Gomperts, R. E. Stratmann, O. Yazyev, A. J. Austin, R. Cammi, C. Pomelli, J. W. Ochterski, R. L. Martin, K. Morokuma, V. G. Zakrzewski, G. A. Voth, P. Salvador, J. J. Dannenberg, S. Dapprich, A. D. Daniels, O. Farkas, J. B. Foresman, J. V. Ortiz, J. Cioslowski, and D. J. Fox, Gaussian 09, Revision D.01; Gaussian, Inc., Wallingford CT, 2013.

[3] H. Buss, T. P. Chan, K. B. Sluis, N. M. Doming, C. C. Winterbourn, Protein Carbonyl Measurement by a Sensitive Elisa Method. Free Radical Bio Med, 1997, 23, 361-366. 
[4] D. Zhang, D. Jiang, M. Yanney, S. Zou, A. Sygula, Ratiometric Raman spectroscopy for quantification of protein oxidative damage. Anal Biochem 2009, 391 (2), 121-6. 